\title{
CADENA DE PRODUCCIÓN SOSTENIBLE EN COSTOS Y SEGURIDAD ALIMENTICIA NACIONAL
}

\author{
CHAIN OF SUSTAINABLE PRODUCTION COSTS AND NATIONAL FOOD SECURITY \\ Elsa Esther Choy Zevallos* \\ Docente Asociada de la Facultad de Ciencias Contables \\ Universidad Nacional Mayor de San Marcos-UNMSM / Lima-Perú \\ [Recepción: Marzo de 2015 / Conformidad: Abril 2015]
}

\section{RESUMEN}

La problemática ambiental existente a nivel mundialy; especialmente en nuestro país, es el resultado del abuso del suelo, tratando de explotarlo sin cuidar su preservación, y utilizando agroquímicos contaminantes que causan daño a la salud, como producto del consumo de alimentos frescos contaminados derivados de los residuos de productos químicos.

Es necesario un nuevo enfoque en la cadena productiva agrícola que garantice un desarrollo sostenible con viabilidad económica, ecológica, justicia social y humanística, incremento en la capacidad de autoconocimiento en las comunidades rurales, donde se integren los procesos naturales con las de producción agrícola, alcanzando mayor productividad de plantas y productos orgánicos. En tal sentido, el estudio trata de responder la siguiente pregunta ¿Cuál es el impacto de implementar un sistema de producción sostenible con la agricultura orgánica?

El objetivo es señalar la importancia de implementar procesos productivos orgánicos como una manera sostenible para mejorar los costos y seguridad alimenticia de la población. Asimismo, plantear la hipótesis: la agricultura orgánica es un sistema de producción sostenible orientada al manejo eficiente de los recursos naturales y conservación de los servicios que brindan los ecosistemas, generando beneficios económicos, sociales y ambientales.

Palabras clave:

Agricultura orgánica; cadena de producción; sostenibilidad; gestión; costos.

\begin{abstract}
Environmental problems exists worldwide; especially in our country, as the result of soil abuse, exploiting it without care for its preservation, and using agrochemicals that damage health, as a result of consuming contaminated fresh food derived from residues of chemical products.

A new approach to agricultural chain production is necessary, to ensure sustainable development with economic viability, environmental sustainability, social justice and humanistic, increased capacity for self-knowledge in rural communities- where natural processes are integrated with agricultural production-reaching for higher productivity of plants and organic products. In this regard, the study seeks to answer the following question: What is the impact of implementing a sustainable production system with organic agriculture?

The goal is to highlight the importance of implementing organic production processes as sustainable way to improve costs and food security of the population. Also we propose the hypothesis: Organic agriculture is a sustainable production system oriented to efficient management of natural resources and conservation of services provided by ecosystems, generating economic, social and environmental benefits.
\end{abstract}

\section{Keywords:}

Organic farming; production chain; sustainability; management; costs.

\footnotetext{
* Doctora en Ciencias Contables y Empresariales, Magister en Dirección Financiera y Contador Público Colegiado.
} Email: eschoyz@outlook.com 


\section{INTRODUCCIÓN}

La Organización de las Naciones Unidas -ONU, señala que para el año 2025 , el $83 \%$ de la población mundial, aproximadamente unas 8.500 millones de personas, vivirán en países en desarrollo. Sin embargo la mayoría de estos países no han logrado la implementación de procesos productivos sostenibles en agricultura y seguridad alimenticia. Es posible que, la capacidad de recursos y tecnologías disponibles no alcance para satisfacer las demandas de alimentos de esta población.

La investigación referida a la Aptitud de Tierras y Oportunidades para el Desarrollo Rural en Perú, Helfgott et al. (2010), señala cifras proyectadas, estimando que la población aumentará hasta 34 millones en 2020 y 40 millones en 2030, el aumento ocurrirá básicamente en los sectores urbanos, mientras que el número de personas en el sector rural será el mismo o disminuirá debido a las migraciones. Por tanto, los productores rurales tendrán que ser eficientes para aumentar la producción de alimentos para su propio consumo y satisfacer la demanda.

En consecuencia, tomando en cuenta los escenarios mundial y nacional, el presente trabajo plantea un nuevo enfoque de cadena productiva reorientada hacia la producción orgánica, en una perspectiva de contribución a la mejora de la competitividad del país, sobre la base de la conservación de los servicios de los ecosistemas. Se trata de reconocer que un sistema de producción de esta naturaleza, impulsa cadenas de valor que estimulan la economía local y permiten participar en mercados de acuerdo con la escala de producción.

Para el cumplimiento del objetivo planteado, se describe un marco teórico desarrollado por diversos autores, relacionado con productos orgánicos, cadena productiva, sostenibilidad y costos. Asimismo, señala las pautas para desarrollar una metodología de cadena productiva, dando prioridad a sistemas de producción de carácter sustentable, donde los participantes tengan bien claro la problemática ambiental existente y puedan establecer explotaciones agrícolas rentables, usando técnicas de producción que no destruyan el ambiente, fáciles de aplicar y accesibles a todo productor desde el punto de vista económico
Esta investigación es relevante para el sector académico, juega un papel prioritario desde los centros de educación superior como la Universidad Nacional Mayor de San Marcos, donde de manera directa o indirecta y, gracias a las políticas y estrategias; la investigación e innovación, y los procesos de capacitación que se desarrollan de forma integrada y multidisciplinaria, generan un gran marco de conocimiento que potencia al sector de producción agrícola y gestión de costos para la seguridad alimenticia de nuestro país.

Los resultados del estudio, orientarán a mejorar la formulación de políticas a favor de la agricultura orgánica en el Perú, en un contexto de competitividad y globalización.

\section{MEDIO AMBIENTE}

La adopción informal por parte de las Naciones Unidas sobre el concepto de desarrollo sostenible parte de la creación en 1983 de la Comisión mundial sobre ambiente y el desarrollo, que en 1987 publicó su informe titulado: "Nuestro futuro común", también es conocido como el Informe Brundtland. Este informe subrayó a la pobreza de los países del sur y al consumismo extremo de los países del norte como las causas fundamentales de la insostenibilidad del desarrollo y la crisis ambiental.

El desarrollo del programa 21, se inició en diciembre de 1989 con la aprobación en la asamblea extraordinaria de las Naciones Unidas en Nueva York de una conferencia sobre el medio ambiente y el desarrollo como fuera recomendada por el informe Brundtland y con la elaboración de borradores del programa y luego de un complejo proceso de revisión, consulta y negociación, culminó con la Conferencia de las Naciones Unidas sobre Medio Ambiente y Desarrollo, conocida como Cumbre de Río o Cumbre de la Tierra, llevada a cabo del 3 al 14 de junio de 1992 en Río de Janeiro, en donde representantes de 179 gobiernos acordaron adoptar el programa.

Después de la primera cumbre histórica de Río de Janeiro en 1992 y diez años después de la de Johannesburgo en 2002, se realizó la Cumbre de la Tierra Río+20, llamada oficialmente Conferencia de Naciones Unidas sobre Desarrollo Sustentable, se celebró del 20 al 22 de junio de 2012 en Río de Janeiro, Brasil. Esta cumbre fue un nuevo intento de Naciones Uni- 
das en el comienzo de milenio para avanzar sobre el compromiso de los Estados y la comunidad mundial en los grandes cambios de este siglo XXI. La ONU invitó a los Estados, la sociedad civil y los ciudadanos a "sentar las bases de un mundo de prosperidad, paz y sustentabilidad" (1987).

En Lima Perú, se realizó del 1 al 12 de diciembre de 2014, la XX Conferencia Internacional sobre Cambio Climático o vigésima Conferencia de las Partes (COP 20). Esta conferencia fue organizada por la Convención Marco de las Naciones Unidas sobre el Cambio Climático. El objetivo de la conferencia fue concluir un acuerdo provisorio mundial para reducir sus emisiones de gases de efecto invernadero, en concordancia a la Declaración sobre los Bosques y el Convenio sobre la Diversidad Biológica.

\section{DESARROLLO SOSTENIBLE}

Del informe titulado, "Nuestro Futuro Común" (1987) de la Comisión Mundial sobre el Medio Ambiente y el Desarrollo, define "el desarrollo sostenible como la satisfacción de las necesidades de la generación presente sin comprometer la capacidad de las generaciones futuras para satisfacer sus propias necesidades". El desarrollo sostenible ha emergido como el principio rector para el desarrollo mundial a largo plazo. Costa de tres pilares, el desarrollo sostenible trata de lograr, de manera equilibrada, el desarrollo económico, el desarrollo social y la protección del medio ambiente.

\section{Agricultura y el problema medio ambiente}

De la información recogida por algunos autores, puede observarse que por muchos años existe interés en mitigar el impacto ambiental y el uso de mejores prácticas en la explotación de bosques y suelo.

La agricultura ha sido la actividad más importante para la supervivencia y el bienestar de la humanidad por lo que constituye una de las principales fuentes de desarrollo y progreso de la sociedad, sin embargo en muchas partes de nuestro planeta, no está cumpliendo con su función vital de alimentar a la población, ofrecer una diversidad de productos y generar entradas estables (García, 1999).

En la mayoría de los círculos agrícolas cientíicos se ha llegado a la percepción general de que la agricultura moderna enfrenta una crisis ambiental. La raíz de esta crisis radica en el uso de práctica agrícolas intensivas basadas en el uso de altos insumos que conllevan a la degradación de los recursos naturales a través de procesos de erosión de los suelos, salinización, contaminación con pesticidas, desertificación, pérdida de fitomasa y por ende reducciones progresivas de la productividad (Altieri, 1994).

El interés mundial por los problemas del medio ambiente, es una realidad, por ello, actuar encaminado hacia su protección, en la actividad agrícola significa disponer de una dirección científicamente proyectada, para de ésta forma satisfacer las necesidades humanas sin afectar el entorno; por ello, la necesidad de un continuo crecimiento agrícola con criterios de alta racionalidad económica y ecológica, que permita mayores beneficios a los productores y un servicio eficiente a la sociedad, en cuanto a seguridad alimentaria y mejora del ambiente, requiere disponer de proyectos que integren todos los componentes del sistema y que permita el pensamiento global y la actuación local como pautas importantes en la definición sistémica del análisis de los ecosistemas agrícolas (FAO,1999).

El desarrollo agrícola futuro requiere de nuevos enfoques, que permitan ampliar las posibilidades de resolver las necesidades siempre crecientes de la población; a su vez es necesario que los sistemas que se utilicen sean sostenibles, desde los puntos de vista: productivo, ecológico, económico, y además sean socialmente justos y culturalmente aceptables (Ortiz y Vera, 2001).

\section{MERCADO DE CULTIVOS ORGÁNICOS}

El Centro de Investigación de la Universidad Pacífico, publicó un documento de discusión DD 12/14, relacionado con La agricultura orgánica: los beneficios de un sistema de producción sostenible, por el cual la autora responsable Gómez, Rosario (Dic. 2012), cita lo siguiente:

\section{Mercado internacional}

En el mundo, el mercado de los productos orgánicos ha crecido considerablemente en los últimos años. Esto debido a una expansión de la demanda explicada por los cambios en los gustos y las preferencias hacia productos inocuos y naturales. En Estados Unidos, las ventas al por menor de alimentos orgánicos han pasado de US\$ 3.6 mil millones en 1997 a US\$ $21.1 \mathrm{mil}$ millones en el 2008 (Dimitri \& Oberholtzer, 2009). En Europa, destaca el mercado alemán, que registró 
ventas por US\$ 7.96 mil millones, y Reino Unido, con US\$2.83 mil millones (FiBL \& IFOAM, 2011).

Con relación a nuestro país, el documento cita algunos datos estadísticos importantes relacionado con los productos orgánicos.

\section{Mercado nacional}

El Perú registra un crecimiento significativo de la producción orgánica. Esta es diversificada, pues comprende más de 73 cultivos distintos y se desarrolla en más de 20 departamentos del país, y tiene como principal destino el mercado de exportación, aunque el consumo interno está creciendo gradualmente, aún es incipiente.

Si bien se tiene una oferta diversificada y descentralizada de productos orgánicos, esta se concentra en cinco productos (café, banano, cacao, quinua y mango), qe explican más del $47 \%$ del valor total de las exportaciones de productos orgánicos. Estos cinco productos registraron un crecimiento en el valor de exportación de $50 \%$ entre el 2010 y el 2011, pasando de US\$ 198.5 millones en el 2010 a US\$ 304.8 millones al año siguiente (Promperú, 2011).

El café lidera el ranking del valor de las agroexportaciones de productos orgánicos, seguido del banano, el cacao, la quinua y el mango. Por su parte, el banano orgánico lidera el mismo ranking pero basado en el volumen exportado. Seguidamente se encuentran el café, el cacao, el mango y la quinua.

Un dato importante recogido de este documento se relaciona con la producción orgánica derivada de la diversidad biológica nativa, como es el caso de la lúcuma, el orégano, sacha inchi, la sangre de grado, la uña de gato y el yacón. (2012).

\section{PRODUCTOS ORGÁNICOS}

Pizarro, N. (2012), señala que los productos orgánicos son aquellos que fueron producidos mediante técnicas no contaminantes. Para que un producto se considere orgánico tiene que cumplir con ciertos requisitos:

- Que no use productos de síntesis química en su producción

- Que realicen prácticas para conservar la tierra y sus nutrientes

- Que cuenten con una certificación vigente avalada con alguna instancia reconocida

- Que use racionalmente los recursos naturales.
En síntesis, son productos limpios de residuos químicos y adecuados para el cuidado del medio ambiente y desarrollo sostenible en seguridad alimenticia.

\section{PRODUCTOS ORGÁNICOS}

La cadena productiva es un conjunto de agentes económicos que participan desde la provisión de insumos, transformación, producción y comercialización hasta el consumo final. Fellner (2004) menciona que:

La actividad agropecuaria pasa por procesos que tiene característica muy diferente a una producción industrial, tales como:

Crecimiento vegetativo: es un proceso productivo biológico autónomo basado en la genética. Este concepto tiene a su vez características propias: Ciclo vegetativo: serie de fenómenos biológicos que suceden una y otra vez hasta la obtención del producto final, y cuya duración y desarrollo es definido por las plantas y animales. Están estrechamente relacionados con el suelo y el clima.

Procesos productivos: es la forma en que las diferentes plantas desarrollan el ciclo productivo. Se puede clasificar en: a) Procesos terminales: son aquellos que se transforman en producto de consumo para el hombre, antes de cumplirse el año del ciclo. b) Procesos continuos: son aquellos que una vez alcanzada la edad adulta, generan los productos que retroalimentan el ciclo. c) Procesos prolongados: son aquellos con ciclos superiores al año para transformarse en productos utilizados para el consumo del hombre.

\section{COSTOS DE LA CADENA PRODUCTIVA ORGÁNICOS}

El documento de discusión DD 12/14, antes citado, establece que la producción agrícola se caracteriza por tener un proceso productivo multiproducto, con una variedad de cultivos que se producen sobre la base de una dotación de recursos naturales y unidad de gestión.

La producción agrícola generalmente no toma en cuenta las consecuencias que pueda tener determinada forma de uso de los factores de producción en el largo plazo (erosión del suelo, contaminación de agua, etc.).

156/ QVIPUIRAMAYOC | Vol. 23(43) 2015 
La agricultura orgánica maneja un portafolio de cultivos que tienen una vinculación en cuanto a la forma de uso de los factores de producción, tanto fijos (tierra) como variables (agua, mano de obra). En este sentido, este tipo de agricultura no solo genera un flujo de bienes y servicios derivados del uso eficiente de los recursos naturales, sino también la forma de uso que hace de ellos tiene consecuencias favorables en el largo plazo. En este sentido, la producción de cultivos orgánicos corresponde a un sistema donde los factores fijos adquieren importancia porque pueden utilizarse en la producción de cualquiera de los cultivos (Ferguson, 1985).

Cabe precisar que la tierra es un factor de producción fijo en extensión, ubicación y características que varían según clima, topografía, entre otros (Miranowski, 1993). Un aspecto clave para el desarrollo agrícola es la diversidad biológica del suelo (agrobiodiversidad), que alude a toda la diversidad dentro y entre especies que se encuentran en los cultivos y crianzas domesticadas, incluyendo también a los parientes silvestres, las especies polinizadoras que interactúan, las pestes, parásitos y otros organismos (Convenio sobre Diversidad Biológica).

La riqueza de especies debajo del suelo es mayor a la que habita en la superficie (Heywood, 1995), aunque gran parte de estos microorganismos son aún desconocidos (Wall, et ál., 2001). De hecho, un estudio estima que solo conocemos el $1 \%$ de las especies de microorganismos del suelo (Turbé, et ál., 2010). Esta diversidad biológica es la base de procesos y servicios ecosistémicos que son esenciales para la formación de suelos, el mantenimiento de su fertilidad, la regulación de ciclo hidrológico y el control de plagas, entre otros (Turbé, et ál., 2010).

En este sentido, el manejo de dicha diversidad biológica permite que la producción agrícola sea sostenible y costo-efectiva. Por tanto, están estrechamente vinculados y su capacidad de apoyarse mutuamente es crecientemente reconocida (European Environment Agency, 2010).

En un sistema de producción multiproducto y mutinsumo es necesario tener en cuenta la relación entre el stock de recursos naturales que subyace al flujo de servicios derivados de los mismos. Varios tienen la propiedad física que si el flujo utilizado en el presente se reduce, el stock aumentará y, por tanto, se tendrá un mayor flujo disponible en el futuro. Ejemplo de ello es el uso de un acuífero a lo largo del tiempo. Si se extrae agua subterránea sin tener en cuenta la capacidad de regeneración del acuífero en el largo plazo, la disponibilidad de agua se reducirá, se incrementarán los costos de extracción y bombeo en el siguiente periodo (por su mayor profundidad) (Howitt, Richard y Robert Taylor, 1993) (2014).

\section{CONCLUSIONES}

1. El bajo rendimiento de la agricultura en muchos países del mundo se atribuye a varios factores, por lo general son: condiciones climatológicas, déficit de recursos e insumos, degradación de los suelos, deficiente logística en las etapas de riego, cultivo y cosecha.

2. El principal objetivo de una cadena productiva agrícola sostenible es utilizar los recursos sin afectar su disponibilidad en el futuro, aumentando la producción de alimentos para mejorar la seguridad alimentaria.

3. Se evidencia que la agricultura orgánica es un sistema de producción sostenible que acompaña muchos beneficios como son económicos, sociales y ambientales.

\section{REFERENCIAS BIBLIOGRÁFICAS}

1. DE CAMINO, R. (2005) El manejo de bosques y plantaciones forestales al 2025 en Costa Rica. Qué podemos esperar? "El sector forestal en Costa Rica, perspectivas al 2025. Costa Rica

2. FELLNER, A. (2004) Pequeño productor agrícola: Informe de costos y aplicación del tablero de control. XXVII Congreso Argentino de Profesores Universitarios de Costos.

3. FRONTI DE GARCIA, L. (2009) Sumideros y mecanismo de desarrollo limpio: una propuesta de desarrollo sostenible para países del MERCOSUR, documentos de trabajo de Contabilidad Patrimonial y Ambiental.

4. SILVA, J. (2011) Sistema de Información Ambiental y Económica Integrada. Ámbito Internacional. ONU.

5. QUINTERO, M. (2006) Modelo de Optimización para Evaluación Ex Ante de Alternativas Productivas y Cuantificación de Externalidades Ambientales en Cuencas Andinas. Proyecto Re- 
gional Cuencas Andinas CONDESAN y Centro Internacional de Agricultura Tropical CIAT, Cali, Colombia.

\section{REFERENCIAS HEMEROGRÁFICAS}

1. Asociación Española de Contabilidady Administración de Empresas. AECA (2011) El Concepto de la Contabilidad Ambiental y la normalización. GestioPolis.

2. Autoridad Autónoma de Cuenca Hidrográfica Chira Piura y PDRS, GTZ (2004) Diagnóstico Participativo con el Enfoque de Gestión del Riesgo de la Cuenca del Río Piura, Piura, Perú.

3. CEDEPAS NORTE y Proyecto Regional Cuencas Andinas. (2007) Estudio Socio- Económico y de los Sistemas de Producción en la Cuenca del Río Jequetepeque, Cajamarca, Perú.

4. Informe de la Comisión Mundial sobre el Medio Ambiente y el Desarrollo (Comisión Brundtland): Nuestro Futuro Común ONU (11/12/1987).

5. Declaración de Río sobre Medio Ambiente y Desarrollo ONU (1992).

6. Quinto programa comunitario de actuación en materia de medio ambiente: hacia un desarrollo sostenible.

7. La Declaración de Hannover de los líderes municipales en el umbral del siglo XXI Agenda 21.
8. Declaración de Johannesburgo sobre el Desarrollo Sostenible Naciones Unidas. (2002).

9. GARCÍA, C. (2012) Cuentas satélite medio ambientales en la contabilidad macroeconómica -Revista Enfoques - Ed. La Ley- N4.

10. GÓMEZ, R. (2012) La agricultura orgánica: los beneficios de un sistema de producción sostenible. Centro de Investigación de la Universidad del Pacífico. Documento de Discusión DD/12/13.

11. HELFGOTT, S., VARGAS, S., GUTIÉRREZ, A. \& SALVATORE, M. (2010) Aptitud de tierras y oportunidades para el desarrollo rural en Perú. El análisis de BEFS para el Perú, I (39) 25-36.

12. MACHIM, M. (2006) Valoración económica de los recursos naturales: Perspectivas a través de los diferentes enfoques de mercado. Revista Futuros. IV (13).

13. PIZARRO, N. (2010) Productos orgánicos.

14. Banco Interamericano de Desarrollo-BID. (2005). Estudio sobre inversión directa en negocios forestales sostenibles - proyecto atn/ np-8323-r. Índice de atracción a la inversión forestal (IAIF) Informe Final - rev. 01. División de administración de recursos naturales y medio ambiente departamento regional de operaciones. Curitiba - Brasil. Abril / 2005. 